\title{
Impacto COVID 19 sobre la situación económica, en una empresa del sector agrícola
}

\section{COVID 19 impact on the economic situation, in a company in the agricultural sector}

\section{Impacto do COVID 19 na situação econômica, em uma empresa do setor agrícola}

\author{
Sheyla Patricia Padilla Gabriel \\ sheylapadilla@upeu.edu.pe \\ iD \\ https://orcid.org/0000-0003-2893-6284 \\ Universidad Peruana Unión \\ Yeni Lisbeth Pérez Huamán \\ yeniperez@upeu.edu.pe \\ iD https://orcid.org/0000-0002-1155-141X \\ Universidad Peruana Unión
}

\author{
Gisela Anabel Vargas Fuentes \\ giselavargas@upeu.edu.pe \\ iD https://orcid.org/0000-0002-6743-2209 \\ Universidad Peruana Unión \\ Rocío Elizabeth Sánchez Vásquez \\ rocioelizabeth@upeu.edu.pe \\ (iD https://orcid.org/0000-0002-8935-3392 \\ Universidad Peruana Unión
}

\author{
Jesús Mariano Gómez Miguel \\ jgomezmiguel1@gmail.com \\ iD https://orcid.org/0000-0003-0706-072X \\ Universidad Nacional Daniel Alcides Carrión
}

Recibido: 06 de enero de 2021

Aceptado: 16 de junio de 2021

\section{Resumen}

El estudio consideró el objetivo de determinar el impacto de la COVID-19 sobre la situación económica de una empresa agrícola, cuyo enfoque es cuantitativo, de tipo descriptivo, comparativo y con un diseño no experimental. Los datos fueron recolectados a través de la técnica de revisión documental, de una muestra de 48 registros contables. Los resultados fueron: 1) la COVID-19 incide de forma negativa sobre las ventas, con un valor de $\mathrm{T}=-1.906$. y un $\mathrm{p}$ valor de 0.083 ; y 2) la COVID-19 incide de forma negativa sobre las compras, con un valor de $\mathrm{T}=-1.897$ y un $\mathrm{p}$ valor de 0.084. 3) La COVID-19 incide de forma negativa sobre las cuentas por cobrar con un valor de $T=-1.764$ y un $p$ 
valor de 0.105 . Se concluyó que la empresa en promedio ha dejado de comprar cada mes durante la pandemia un monto de 95662.015 soles; no ha significado un cambio significativo en la economía de la empresa; respecto de las ventas se concluyó que la empresa ha dejado de percibir en promedio 39024.41 soles; no trajo mayores consecuencias en la economía de la empresa; respecto de las cuentas por cobrar, se concluyó que la empresa ha percibido un $0.30 \%$ más de deudas de los clientes.

Palabras clave: Incidencia de COVID, ventas, compras.

\section{Abstract}

The study considered the objective of determining the impact of COVID-19 on the economic situation of an agricultural company, whose approach is quantitative, descriptive, comparative and with a non-experimental design. The data were collected through the document review technique, from a sample of 48 accounting records. The results were: 1) COVID-19 has a negative impact on sales, with a value of $\mathrm{T}=-1,906$. and a $p$ value of 0.083; and 2) COVID-19 has a negative impact on purchases, with a value of $\mathrm{T}=-1.897$ and a $\mathrm{p}$ value of 0.084. 3) COVID-19 has a negative impact on accounts receivable with a value of $T=-1.764$ and a $p$ value of 0.105 . It was concluded that the company, on average, has stopped buying an amount of 95,662,015 soles each month during the pandemic; it has not meant a significant change in the economy of the company; Regarding sales, it was concluded that the company has stopped receiving an average of 39024.41 soles; It did not bring major consequences in the economy of the company; Regarding accounts receivable, it was concluded that the company has received $0.30 \%$ more debts from customers.

Keywords: COVID incidence, sales, purchases.

\section{Resumo}

O estudo teve como objetivo determinar o impacto do COVID-19 na situação económica de uma empresa agrícola, cuja abordagem é quantitativa, descritiva, comparativa e com desenho não experimental. Os dados foram coletados por meio da técnica de revisão documental, a partir de uma amostra de 48 registros contábeis. Os resultados foram: 1) COVID-19 tem um impacto negativo nas vendas, com um valor de $\mathrm{T}=-1,906$. e um valor de $\mathrm{p}$ de 0,083; e 2) COVID-19 tem um impacto negativo nas compras, com um valor de $\mathrm{T}=-1,897$ e um valor de $\mathrm{p}$ de 0,084. 3) COVID-19 tem um impacto negativo nas contas a receber com um valor de $\mathrm{T}=-1,764$ e um valor de $\mathrm{p}$ de 0,105 . Concluiu-se que a 
empresa, em média, deixou de comprar 95.662.015 soles por mês durante a pandemia; não significou uma mudança significativa na economia da empresa; Em relação às vendas, concluiu-se que a empresa deixou de receber em média 39024,41 soles; Não trouxe grandes consequências na economia da empresa; Em relação ao contas a receber, concluiu-se que a empresa recebeu $0,30 \%$ mais dívidas de clientes.

Palavras-chave: Incidência COVID, vendas, compras.

\section{Introducción}

En el mundo comercial, el hecho de que una empresa goce de buena economía genera en la misma un crecimiento adecuado. Aquellas empresas que no cuentan con estabilidad económica, enfrentan problemas asociados al desarrollo saludable de la actividad económica. Sin embargo, la situación que sufre hoy el mundo, no permite que las empresas dispongan actividades económicas estables.

En consecuencia de la crisis económica por la pandemia, las empresas enfrentan diferentes problemas. Según el Instituto Nacional de Estadística e Informática INEI (2020), la producción nacional del sector agrícola, decreció en 18,06\%, por la falta de demanda en el mercado productivo. Es importante tener en cuenta cuáles pueden ser los efectos del problema: crisis, quiebra, liquidación, reducción de empleados y, a la vez, la generación de un problema más para la socioeconomía. Según Radio Hotelería y Turismo, un $60 \%$ de los negocios cobrará menos del 25\% de sus cuentas. Según Radio Programas del Perú RPP (2021), un 75\% tendría problemas para cobrar sus cuentas. En los comerciantes agrícolas, se han observado dificultades, la falta de demanda de los productos, problemas para transportar la mercadería, inconvenientes para cobrar a los clientes, trabajadores infectados, etc. Esta investigación beneficiará a la empresa Agrofer Los Andes, ya que se obtendrá conocimiento de los sucesos transcurridos por la situación del COVID-19. Además, observar cómo la empresa puede mejorar y responder ante futuros problemas similares a lo acontecido.

El estudio obtendrá información eficaz y detallada, con el propósito de conocer el impacto económico que ha sufrido el sector agrícola, en consecuencia de la pandemia, generando un beneficio para los usuarios interesados en mejorar la economía del sector agrícola, también para futuros investigadores, quienes quieran realizar estudios de mayor profundidad.

Según Chang y Camelli (2020), su investigación tuvo el objetivo: analizar cómo América Latina afronta la COVID-19. Esta investigación es descriptiva, de método 
inductivo, de corte transversal y de tipo no experimental. Llegaron a la conclusión de que Latinoamérica tuvo, en consecuencia de la pandemia, un menor crecimiento económico, por lo que afrontaría la pandemia con inferiores herramientas, específicamente con limitaciones enormes en infraestructuras sanitarias y estancamiento económico.

Gutierrez (2021), por su parte, construye su objetivo: proponer la implementación de un Plan de Gestión Logística, para disminuir el impacto generado por la COVID -19, en la empresa Agrovisión Perú SAC. Esta investigación es no experimental, transversal y tipo básica - descriptiva; el instrumento utilizado fue el cuestionario. La conclusión a la que llegó es que el impacto de la COVID-19 en la operación de la empresa se evidencia, ya que al aplicar las herramientas de diagnóstico se enlistan una serie de causas directamente determinadas por la COVID-19, tanto en el entorno, así como en la compañía. Se plantea una propuesta de mejora orientada para atacar directamente los efectos de la COVID-19 en la empresa, con el uso de herramientas de gestión de inventarios y aprovisionamiento.

Fernández (2021), en su tesis, aborda el objetivo: identificar el impacto económico y social de la crisis causada por la COVID-19 en el Perú. Su investigación es de tipo descriptivo-explicativo y de enfoque cuantitativo, el diseño es no experimental y transeccional. Generaron la conclusión de que esta incertidumbre no debe frenar las acciones del Gobierno, cuyos responsables deben elaborar y ejecutar políticas públicas, fiscales y monetarias expansivas, además presentar mejores fundamentos macroeconómicos, impulsando las inversiones públicas y privadas (exportaciones e importaciones), a fin de incentivar el sistema empresarial. Consecuentemente, los tres niveles del Gobierno, así como las instituciones públicas y privadas, deberán planificar acciones o medidas de carácter político, económico y social, buscando un equilibrio entre los protocolos sanitarios y el reinicio eficiente de operaciones financieras, para lograr una recuperación adecuada de la actividad económica.

\section{Metodología}

\section{Diseño metodológico}

El diseño de la investigación es descriptivo y comparativo. Es descriptivo, porque se describen los datos obtenidos en los registros de compras y ventas de la empresa, incluyendo tablas y datos estadísticos. También es comparativo, porque los datos fueron recolectados durante un periodo determinado y en diferentes situaciones. Su diseño es no experimental y longitudinal. No experimental, porque no se realizó ningún tipo de 
manipulación de los datos; longitudinal, porque se realizó mediciones en un tiempo definido, analizando cambios económicos antes y durante pandemia. La técnica es no probabilística, por conveniencia, ya que, según Hernández y Mendoza (2018), las investigaciones con esta técnica se realizan con datos a los que se tiene acceso disponible.

\section{Diseño muestral}

La muestra está constituida por 24 registros de compras y 24 registros de ventas de la empresa "Agrofer los Andes S.A.C", estos registros corresponden a los años 2019 y 2020; de esta manera, se analiza el impacto de la empresa antes y durante pandemia.

\section{Técnicas de recolección de datos}

La recolección de datos cumple con 3 características, según Fernandez, Baptista y Hernandez (2014), deben ser confiables, válidos y objetivos. Este estudio de la recolección de datos se realizó en EXCEL, y se analizó a profundidad en el programa estadístico SPSS, con la finalidad de agilizar la información, para tener datos organizados y concisos. De esta manera, obtendremos resultados exactos, que nos darán a conocer porcentajes del impacto en las compras y ventas 2019 y 2020 de la empresa.

\section{Técnicas estadísticas para el procesamiento de la información}

El estudio es parte del proceso, permite generar respuesta a los objetivos; usa la estadística inferencial. Primero, se analiza la normalidad de las variables a través del coeficiente del Shapiro Wilk; posteriormente, se aplica la prueba paramétrica o no paramétrica que serán T-student o la prueba de Wilcoxon, para muestras relacionadas respectivamente. Para esto se usa el programa estadístico SPSS.

\section{Aspectos éticos}

Teniendo en cuenta la confianza de la empresa en los investigadores, el primer punto ético es recalcar que no se vulnera la privacidad de la empresa. Para realizar este proyecto también respetó el reglamento del centro de estudios; por último, también consideró alinearse a las exigencias del APA 7ma edición, y así entregar una buena investigación. 


\section{Resultados}

\section{Análisis de normalidad}

Tabla 1

Prueba de normalidad de los ingresos

\begin{tabular}{llll}
\hline & $\begin{array}{l}\text { Shapiro-Wilk } \\
\text { Estadístico }\end{array}$ & gl & p valor \\
\hline Ventas en pandemia & 0.948 & 12 & 0.612 \\
Ventas sin pandemia & 0.969 & 12 & 0.900 \\
Compras en pandemia & 0.875 & 12 & 0.076 \\
Compras sin pandemia & 0.968 & 12 & 0.883 \\
Cuentas por cobrar en pandemia & 0.913 & 12 & 0.235 \\
Cuentas por cobrar sin pandemia & 0.981 & 12 & 0.986 \\
\hline
\end{tabular}

De acuerdo con el análisis de normalidad, se observa que el total de las variables estudiadas obtuvieron resultados superiores a 0.05 ; es decir, la distribución de las variables en su total es normal. Considerando esto, las pruebas para medir el impacto será la prueba de T de Student.

Tabla 2

Estadísticos descriptivos de los ingresos

\begin{tabular}{ccccc}
\hline & Media & $\mathrm{N}$ & $\begin{array}{c}\text { Desv. } \\
\text { Desviación }\end{array}$ & $\begin{array}{c}\text { Desv. Error } \\
\text { promedio }\end{array}$ \\
\hline Ventas en pandemia & 389693.07 & 12 & 80332.5606 & 23190.0127 \\
Ventas sin pandemia & 428717.49 & 12 & 64083.9948 & 18499.4558 \\
\hline
\end{tabular}

Se observa que la media de las ventas ha sido de 389,693.07, durante los 12 primeros meses de la pandemia por COVID 19, con una desviación estándar de 80,332.56. También se observa que la media de las ventas, en un periodo de 12 meses previos a la pandemia, ha sido de 428,717.42 con una desviación estándar de 64,083.99. 
Tabla 3

Diferencia de medias de los ingresos

\begin{tabular}{|c|c|c|c|c|c|}
\hline & \multicolumn{3}{|c|}{ Diferencias emparejadas } & \multirow[b]{2}{*}{$\mathrm{t}$} & \multirow[b]{2}{*}{$\mathrm{p}$ valor } \\
\hline & Media & $\begin{array}{c}\text { Desv. } \\
\text { Desviación }\end{array}$ & $\begin{array}{l}\text { Desv. Error } \\
\text { promedio }\end{array}$ & & \\
\hline $\begin{array}{l}\text { Ventas en } \\
\text { pandemia }\end{array}$ & & & & & \\
\hline $\begin{array}{c}\text { - Ventas } \\
\text { sin } \\
\text { pandemia }\end{array}$ & -39024.412 & 70923.168 & 20473.755 & -1.906 & 0.083 \\
\hline
\end{tabular}

\begin{tabular}{|c|c|}
\hline $\begin{array}{c}\square \text { Diferencia de medias de los } \\
\text { ingresos }\end{array}$ & $-39,024.41$ \\
\hline
\end{tabular}

La diferencia de medias entre las ventas en pandemia y ventas sin pandemia es de $-39,024.41$, con una desviación estándar de 70,923.16. Por otro lado, se observa que el valor de $\mathrm{p}$ es de 0.083 ( $\mathrm{p}>0.05$ ), lo que indica que la política de reactivación económica no influyó de forma significativa sobre las compras, en una empresa del sector agrícola en la región de San Martín.

Tabla 4

Estadísticos descriptivos de los egresos

\begin{tabular}{lllll}
\hline & Media & N & $\begin{array}{l}\text { Desv. } \\
\text { Desviación }\end{array}$ & $\begin{array}{l}\text { Media de Error } \\
\text { estándar }\end{array}$ \\
\hline Compras en pandemia & 401442.41 & 12 & 130727.6023 & 37737.80819 \\
Compras sin pandemia & 497104.43 & 12 & 74039.86208 & 21373.46715 \\
\hline
\end{tabular}

Se observa que la media de las compras ha sido de 401,442.41, durante los 12 primeros meses de la pandemia por COVID 19, con una desviación estándar de 130,727.60. También se observa que la media de las compras, durante un periodo de 12 
meses previos a la pandemia, ha sido de 497,104.42 con una desviación estándar de $74,039.86$.

Tabla 5

Diferencia de medias de los egresos

\begin{tabular}{llllll}
\hline & \multicolumn{2}{l}{ Diferencias emparejadas } & & p valor \\
\cline { 2 - 4 } & Media & $\begin{array}{l}\text { Desv. } \\
\text { Desviación }\end{array}$ & $\begin{array}{l}\text { Media de error } \\
\text { estándar }\end{array}$ & $\mathrm{t}$ & \\
\hline $\begin{array}{l}\text { Compras en } \\
\text { pandemia }-\end{array}$ & -95662.015 & 174657.816 & 50419.369 & -1.897 & 0.084 \\
$\begin{array}{l}\text { Compras sin } \\
\text { pandemia }\end{array}$ & & & & \\
\hline
\end{tabular}

La diferencia de medias entre las compras en pandemia y compras sin pandemia es de $-95,662.01$, con una desviación estándar de 174,657.81. Por otro lado, se observa que el valor de p es de 0.084 ( $p>0.05$ ), lo que indica que la política de reactivación económica no influyó de forma significativa sobre las compras, en una empresa del sector agrícola en la región de San Martín.

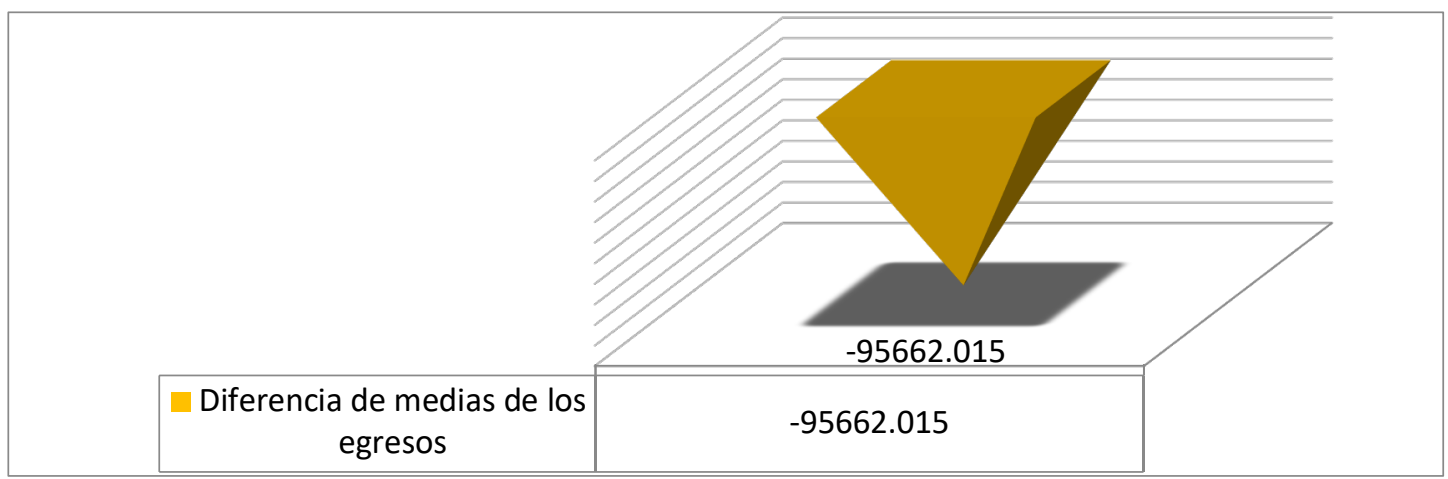

Tabla 6

Estadísticos descriptivos de las cuentas por cobrar

\begin{tabular}{lccll}
\hline & Media & $\mathrm{N}$ & $\begin{array}{l}\text { Desv. } \\
\text { Desviación }\end{array}$ & $\begin{array}{l}\text { Desv. Error } \\
\text { promedio }\end{array}$ \\
\hline $\begin{array}{l}\text { Cuentas por cobrar } \\
\text { en pandemia }\end{array}$ & $3.05 \%$ & 12 & $0.42 \%$ & $0.12 \%$ \\
$\begin{array}{l}\text { Cuentas por cobrar } \\
\text { sin pandemia }\end{array}$ & $2.75 \%$ & 12 & $0.36 \%$ & $0.11 \%$ \\
\hline
\end{tabular}

Se observa que la media de las cuentas por cobrar ha sido de un $3.05 \%$, durante los 12 primeros meses de la pandemia por COVID 19, con una desviación estándar de $0.41 \%$. 
También se observa que la media de las compras, durante un periodo de 12 meses previos a la pandemia, ha sido de $2.75 \%$ con una desviación estándar de un $0.36 \%$.

Tabla 9

Diferencia de medias de las cuentas por cobrar

\begin{tabular}{llllll}
\hline & \multicolumn{3}{c}{ Diferencias emparejadas } & & \\
\cline { 2 - 4 } & Media & $\begin{array}{l}\text { Desv. } \\
\text { Desviación }\end{array}$ & $\begin{array}{l}\text { Desv. Error } \\
\text { promedio }\end{array}$ & t & p valor \\
\hline $\begin{array}{l}\text { Cuentas por cobrar en } \\
\text { pandemia - Cuentas } \\
\text { por cobrar sin } \\
\text { pandemia }\end{array}$ & $0.30 \%$ & $0.59 \%$ & $0.17 \%$ & 1.764 & 0.105 \\
\hline
\end{tabular}

La diferencia de medias entre las cuentas por cobrar en pandemia y cuentas por cobrar sin pandemia es de $0.30 \%$, con una desviación estándar de $0.59 \%$. Por otro lado, se observa que el valor de $\mathrm{p}$ es de 0.105 ( $\mathrm{p}>0.05)$, lo que indica que la política de reactivación económica no influyó de forma significativa sobre las cuentas por cobrar, en una empresa del sector agrícola en la región de San Martín.

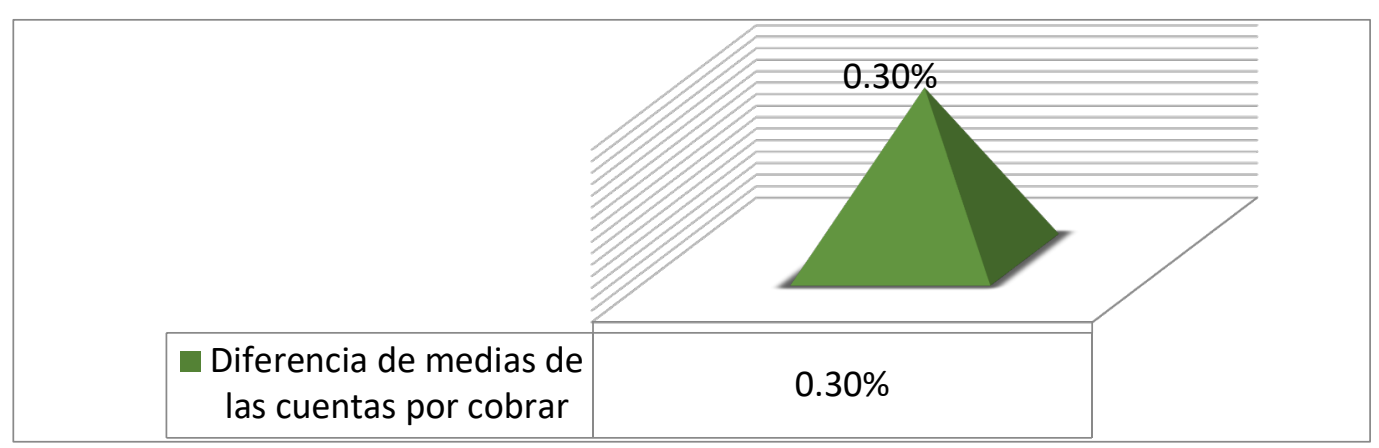

\section{Discusión}

El propósito principal de la investigación es determinar el impacto del COVID-19 sobre la situación económica, en una empresa agrónoma.

En relación con la hipótesis general, la COVID-19 incide de forma significativa sobre la situación económica de una empresa agrónoma. AGRONET (2020), en su investigación, llegó a la conclusión de que al llegar la COVID-19 los planes de avance de las empresas agrícolas se detuvieron, también existieron variaciones en los precios de los productos, creando un efecto negativo en el mercado. 
Según el análisis correspondiente a la primera hipótesis específica, la incidencia de la COVID-19 es de forma significativa sobre los ingresos de una empresa agrícola. Campbell y Fransi (2021), en su investigación, concluyen que la pandemia generó gran cambio, en la manera como realizar las ventas en las empresas; concluyeron también que la COVID-19 causó una gran caída en la actividad comercial de los diferentes rubros y en especial en las ventas de éstas.

Luego del análisis de los datos correspondientes a tercera hipótesis específica, la incidencia de la COVID-19 se da en forma significativa sobre los egresos de una empresa agrícola. Gualán (2020), en su investigación, analiza las compras de las empresas frente al impacto COVID-19; concluye que existe un antes y después en el comportamiento de compra, durante este tiempo de pandemia se experimentaron restricciones financieras, inestabilidad laboral, provocando la limitación de compras.

Hecho el análisis correspondiente a la segunda hipótesis específica, se determina la incidencia de la COVI-19, en forma significativa, sobre las cuentas por cobrar de una empresa agrícola. Puma (2021), luego de finalizar su investigación, concluye que existen problemas financieros en los diferentes rubros, principalmente generando las deudas de los clientes, exponiendo riesgos de cerrar sus puertas al público.

\section{Conclusiones}

Respecto del primer objetivo específico, se concluye que la COVID-19 incide en forma negativa y no significativa sobre las ventas, con una diferencia de medias de 39024.41 soles, con un valor de $\mathrm{T}=-1.906$ y un $\mathrm{p}$ valor de 0.083 . Esto quiere decir que, en el sector agrónomo, una empresa ha dejado de percibir un promedio de -39 024.41 soles de forma mensual, durante el tiempo de la pandemia, además se concluye que esta incidencia no ha sido importante dentro de la empresa.

Respecto del segundo objetivo específico, se concluye que la COVID-19 incide en forma negativa y no significativa sobre las compras, con una diferencia de medias de 95662.01 soles, con un valor de $\mathrm{T}=-1.897$ y un $\mathrm{p}$ valor de 0.084. Es decir, en el sector agrónomo, una empresa ha dejado de comprar su mercadería, con un valor de -95662.01 soles de forma mensual durante el tiempo de la pandemia, además se concluye que esta incidencia no ha sido importante dentro de la empresa.

En referencia del tercer objetivo específico, se concluye que la COVID-19 incide en forma positiva y no significativa sobre las cuentas por cobrar, con una diferencia de medias de $0.30167 \%$, con un valor de $\mathrm{T}=1.764$ y un $\mathrm{p}$ valor de 0.105 . Vale decir que, en 
el sector agrónomo, una empresa ha percibido un $0.30167 \%$ de forma mensual, durante el tiempo de la pandemia, además se concluye que esta incidencia no ha sido importante dentro de la empresa.

\section{Referencias bibliográficas}

AGRONET. (2020). La COVID-19 y su impacto en el modelo de negocio de la agricultura del futuro. https://www.agronet.gov.co/Noticias/Paginas/La-COVID19-y-su-impacto-en-el-modelo-de-negocio-de-la-agricultura-del-futuro.aspx

Campbell, J. B., \& Fransi, E. C. (2021). La dirección comercial en época de pandemia: el impacto del covid-19 en la gestión de ventas. Informacion Tecnologica, 32(1), 10. https://doi.org/10.4067/S0718-07642021000100199

Chang, Camelli, P. (2020). Pandemia COVID-19: Situación política - económica y consecuencias sanitarias en América Latina. Ciencia Unemi, 13(33), 120-128. http://ojs.unemi.edu.ec/index.php/cienciaunemi/article/view/1118/1099

Fernandez; Baptista;Hernandez. (2014). METODOLOGÍA DE LA INVESTIGACIÓN. In Metodología de la investigación. http://observatorio.epacartagena.gov.co/wpcontent/uploads/2017/08/metodologia-de-la-investigacion-sextaedicion.compressed.pdf

Gualán, Q. Á. (2020). Análisis del comportamiento de compra frente a la pandemia Covid-19. Angewandte Chemie International Edition, 1, 14.

Gutierrez. (2021). INDUSTRIAL PLAN DE GESTIÓN LOGÍSTICA PARA DISMINUIR EL IMPACTO GENERADO POR LA COVID-19. 93. https://repositorio.uss.edu.pe/bitstream/handle/20.500.12802/8141/Gutierrez Oliva $\% 2 \mathrm{C}$ Jhonatan Junior.pdf?sequence $=1 \&$ is Allowed $=\mathrm{y}$

Hernández, \& Mendoza. (2018). Metodologìa de la Investigaciòn. Las rutas Cuantitativa Cualitativa y Mixta. In Metodología de la investigación. Las rutas cuantitativa, cualitativa y mixta.

Instituto Nacional de Estadística e Informática INEI. (2020). Panorama Economico Departamental. In Junio (Vol. 2). 
http://m.inei.gob.pe/media/MenuRecursivo/boletines/08-informe-tecnicon08_panorama economico-departamental-jun-2020.pdf

Puma, D. (2021). FUENTES DE FINANCIAMIENTO Y SU INCIDENCIA EN EL DESARROLLO DE LAS MYPES RUBRO FERRETERIAS EN LA PANDEMIA DEL CORONAVIRUS. Universidad Privada San Carlos-Puno, 1, 86. http://repositorio.upsc.edu.pe/bitstream/handle/UPSC/4399/Ronald_Baroni_CHEC ALLA_CARBAJAL.pdf?sequence $=1 \&$ isAllowed $=\mathrm{y}$

Radio Programas del Perú RPP. (2021, February 1). Un 75\% de empresas no podrían pagar sus deudas a tiempo. Andina. Retrieved from https://rpp.pe/economia/economia/un-75-de-empresas-no-podrian-pagar-susdeudas-a-tiempo-coronavirus-en-peru-covid-19-noticia-1318245?ref=rpp

Roxana, J., \& Fernández, H. (2021). Impacto Económico Y Social De La Covid-19 En el Perú. 2, 12. http://recide.caen.edu.pe/index.php/Recide/article/view/49/39 